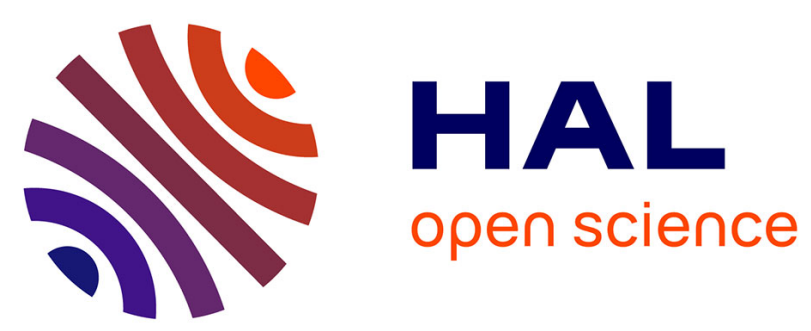

\title{
The impact of a local perturbation on global properties of a turbulent wake
}

Vladimir Parezanović, Olivier Cadot

\section{To cite this version:}

Vladimir Parezanović, Olivier Cadot. The impact of a local perturbation on global properties of a turbulent wake. Physics of Fluids, 2009, 21, pp.071701. 10.1063/1.3184615 . hal-01289971

\section{HAL Id: hal-01289971 \\ https://hal-ensta-paris.archives-ouvertes.fr/hal-01289971}

Submitted on 27 Nov 2017

HAL is a multi-disciplinary open access archive for the deposit and dissemination of scientific research documents, whether they are published or not. The documents may come from teaching and research institutions in France or abroad, or from public or private research centers.
L'archive ouverte pluridisciplinaire $\mathbf{H A L}$, est destinée au dépôt et à la diffusion de documents scientifiques de niveau recherche, publiés ou non, émanant des établissements d'enseignement et de recherche français ou étrangers, des laboratoires publics ou privés. 
Vladimir Parezanović and Olivier Cadot

Unité de Mécanique, Ecole Nationale Supérieure de Techniques Avancées, Paris Tech,

Chemin de la Hunière, 91761 Palaiseau Cedex, France

\section{The impact of a local perturbation on global properties of a turbulent wake}

Stationary perturbation techniques are used to investigate the sensitivity of the global properties of the wake behind a bluff body at moderate Re. The entire recirculation bubble is found to be a sensitive region for the global frequency selection and the quality of the synchronization. A striking position is found at its center where synchronization is destroyed and the frequency is increased. High speed particle image velocimetry sheds some light into the underlying mechanisms, which are interpreted in terms of vorticity interaction, flow reattachment, and flow deviation. Strong mean flow modifications are observed to correspond to drastic changes in drag and its fluctuations.

The effects of a local perturbation on the global mode of two-dimensional (2D) wakes ${ }^{1-3}$ has received much attention since the theoretical work of Giannetti and Luchini. ${ }^{4}$ For Reynolds number close to the first instability, the computation of the direct and adjoint global modes of the basic flow is able to retrieve the spatial structure of the sensitivity of the Bénard-von Kármán instability, obtained experimentally by Strykowski and Sreenivasan. ${ }^{1}$ In the experiment, the sensitivity is deduced from the response of the global mode around the critical Reynolds number to a perturbation created by a small control cylinder. Recent theoretical developments ${ }^{5,6}$ have shown that the basic flow modification introduced by the local perturbation represents the dominant contribution to the global mode sensitivity. The steady disturbance technique is also very successful at large Reynolds numbers to change the wake frequency and reduce the aerodynamics forces. ${ }^{7,8}$ Although the underlying mechanisms for these effects still remain unclear, the possibilities for practical applications are numerous. Using the same technique of stationary perturbations, we investigate the nature of mechanisms responsible for the modification of global properties of a large Reynolds number wake. The area explored with the control cylinder entirely covers the recirculation bubble with high spatial resolution. This enables us to discern regions of the wake, where different mechanisms assume the dominant role. We use high speed particle image velocimetry (PIV) measurements to identify these processes through vorticity dynamics of the wake. The present results are compared to the previous sensitivity analysis ${ }^{1,5,6}$ performed around the critical Reynolds number.

The wake is produced by a symmetric cylinder (that will be referred to as the main cylinder) with a leading edge profiled as a semicircle with a diameter of $D=25 \mathrm{~mm}$ and a flat trailing edge at a right angle to the flow (Fig. 1). The shape has been chosen to avoid the displacement of the separation points due to the presence of the control cylinder (see Ref. 8 for a detailed description of this flow). The primary cylinder is placed in an Eiffel-type wind tunnel and blown over a

\footnotetext{
${ }^{a)}$ Electronic mail: vladimir.parezanovic@ensta.fr.
}

spanwise length of $400 \mathrm{~mm}$, while its total span is $600 \mathrm{~mm}$. On both ends of the primary cylinder, the main velocity decreases to zero through a $1.5 \mathrm{~cm}$ thick boundary layer. The main velocity is $7.5 \mathrm{~m} / \mathrm{s}$ and the Reynolds number of the flow is $\operatorname{Re}=U D / \nu \sim 12500$. The secondary (control) cylinder has a circular cross section with diameter of $d=3 \mathrm{~mm}$ and is mounted on a 2D microcontrol displacement console. Its total span is $800 \mathrm{~mm}$. Displacement is performed in 110 steps of $0.5 \mathrm{~mm}$ in the $y$ direction and 45 steps of $1 \mathrm{~mm}$ in the $x$ direction and covers an area located at the rear of the main body (Fig. 1). For each of the $110 \times 45$ steps, we perform simultaneous, $30 \mathrm{~s}$, measurements of local pressure around the main cylinder and local velocity in the wake. A complete experiment takes about 2 days. For pressure measurements, the main cylinder is equipped with 32 pressure taps at its central section connected to two Scanivalve DSA $3217 / 16 \mathrm{px}$ devices. The form drag $C_{D}$ is then classically estimated by integrating the projected pressure stress over the cylinder perimeter. A hot wire anemometer, sensitive to the modulus of the velocity in the $(x, y)$ plane, is placed at the spanwise center, $6 D=150 \mathrm{~mm}$ downstream of the trailing edge of the main cylinder and level with its upper edge. Autopower spectra of the velocity time series are averaged over windows of $4 \mathrm{~s}$ and have a resolution of $0.25 \mathrm{~Hz}$. The spectra exhibit a strong peak at the vortex shedding frequency from which the mean frequency $f$ (referred to as the global mode frequency) and the width $\Delta f$ of the peak are extracted. The measured quantities are the global mode frequency $f$, the quality factor of the peak $Q=f / \Delta f$, the mean form drag $C_{D}$, and its rate of fluctuations $\delta_{D}=C_{D}^{\text {rms }} / C_{D}$. The natural wake without the control cylinder is taken as the reference case for which all corresponding quantities are indexed with 0: $f_{0}=74.1 \mathrm{~Hz}, Q_{0}=35.7, C_{D 0}=0.76$, and $\delta_{D 0}$ $=0.028$. The measured quantities $m$ are nondimensionalized by taking the difference relative to the reference case $[\tilde{m}$ $\left.=\left(m-m_{0}\right) / m_{0}\right]$ and are mapped as a function of the control cylinder's position in Fig. 2. For all of these maps, red corresponds to an increase $(\tilde{m}>0)$ and blue to a decrease $(\tilde{m}$ $<0)$ in values. For five locations of the control cylinder, labeled as configurations 1-5 in Fig. 2(a) and indicated in 


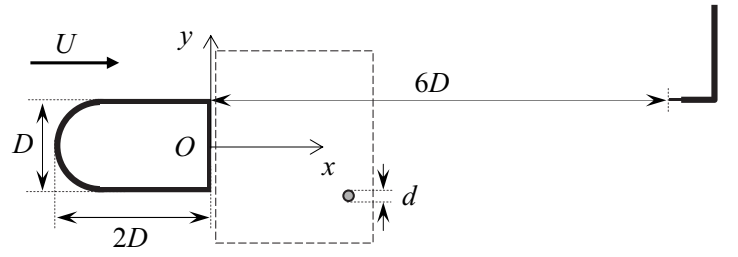

FIG. 1. Sketch of the experimental layout at the spanwise center section $(D=25 \mathrm{~mm}, d=3 \mathrm{~mm})$. The dashed rectangle represents the area explored by the control cylinder. The hot wire probe is depicted in the right hand side of the image.

other maps, high speed PIV acquisitions of 500 fields/s are performed. In order to emphasize the coherent structure dynamics, participating in the global mode, velocity fields are phase averaged over 10 cycles. The PIV results are presented in Fig. 3 as color maps of the phase-averaged vorticity superimposed on a contour plot of the mean vorticity and the zero isoline of the mean longitudinal velocity $\bar{u}=0$ to localize the backflow region.

The $\tilde{f}$-map is shown in Fig. 2(a). We can see global frequency changes in several distinct regions. Although the probe is not placed on the axis of symmetry $O x$ of the bluff body, the resulting map is symmetric. This shows that the frequency of the wake is space independent and is still gov- erned by a global mode. The main causes of the frequency changes are revealed by the PIV measurements and essentially consist of three mechanisms: vorticity interaction, flow deviation, and boundary layer reattachment. The vorticity interaction mechanism is clearly observable for locations 1 [Fig. 3(b)] and 3 [Fig. 3(f)] of the control cylinder. In configuration 1 , the positive vorticity created by the primary cylinder is canceled by the negative one created by the control cylinder. This can be seen in the mean vorticity contours in Fig. 3(b). The positive vorticity in the lower sheet is then reduced while the upper sheet remains unaffected. The coupling between them both, through the Biot and Savart law responsible for vortex shedding, ${ }^{9}$ is then weakened and the global frequency is reduced $(\tilde{f}<0)$ [see location 1 in Fig. 2(a)]. In configuration 3 [Fig. 3(f)], the positive vorticity created by the control cylinder feeds the positive vorticity sheet created by the primary cylinder. Similarly as before, this constructive interaction leads to an increase in the global frequency $(\tilde{f}>0)$ [see location 3 in Fig. 2(a)]. The fact that regions with large positive $\tilde{f}$ extend up to $1 D$ downstream from the body (the bubble length with no control cylinder is $0.84 D$ ) is a consequence of the reattachment of the primary vorticity sheet on the control cylinder. It is due to the entrainment velocity from the low velocity region inside the bubble
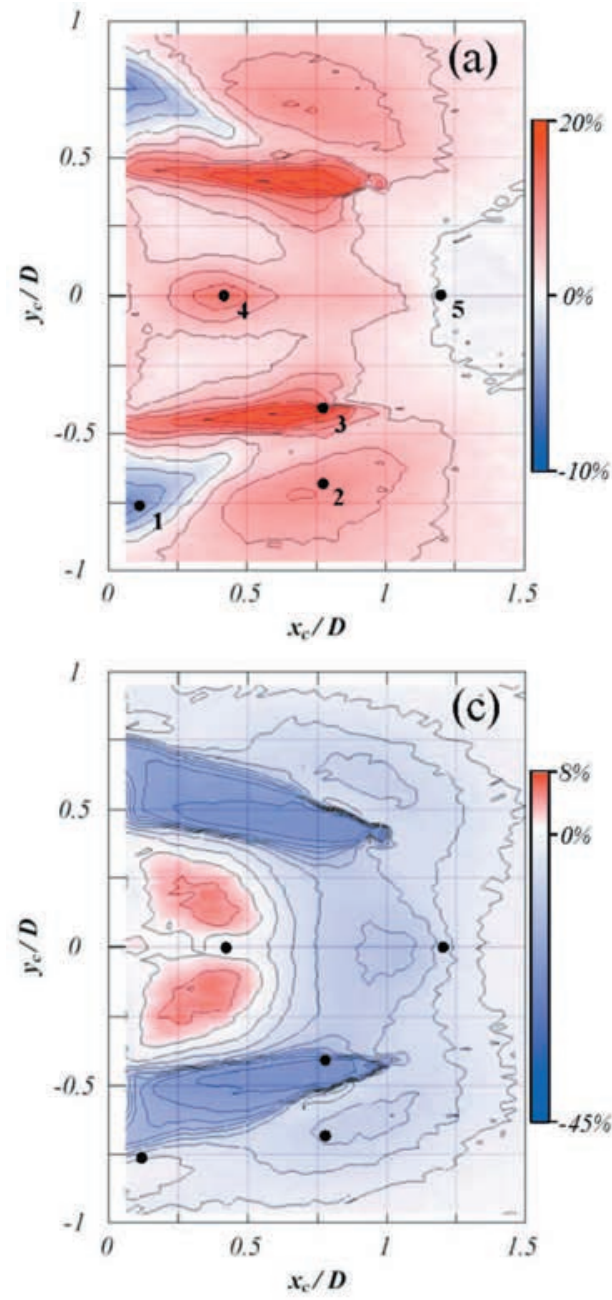
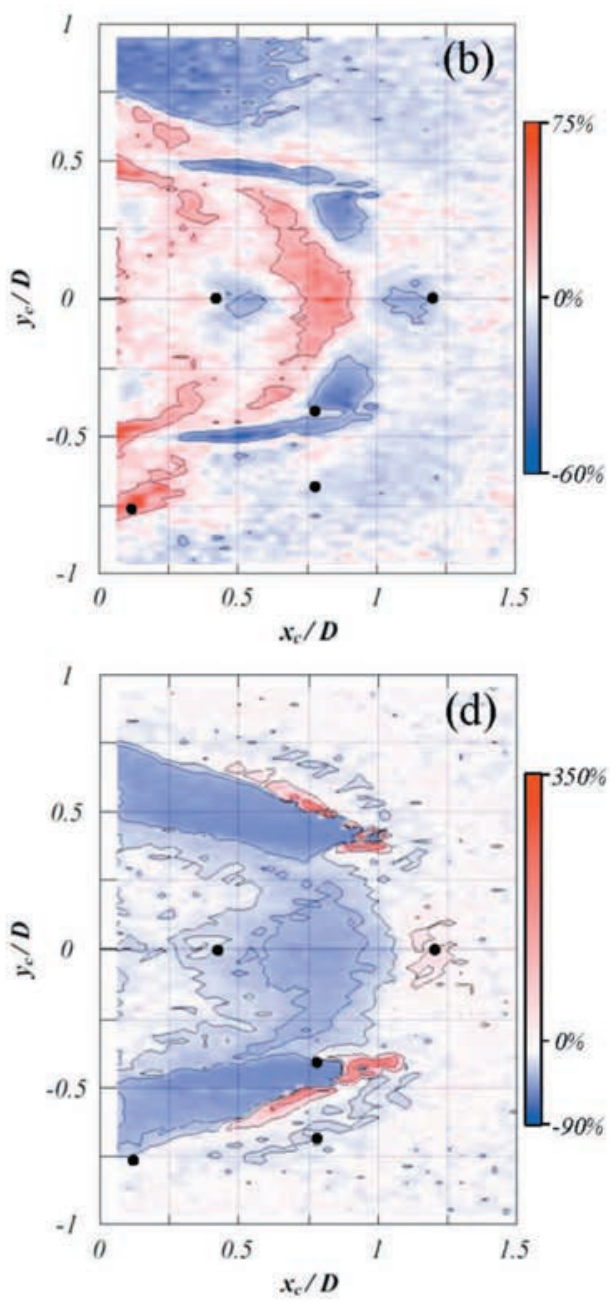

FIG. 2. (Color) Sensitivity of the global properties relative to the natural case (see text). Blue and red are, respectively, negative and positive values, white is zero. (a) Global frequency sensitivity displayed as $\tilde{f}$-map; the interval between the isolines is $3 \%$ with values ranging from $-10 \%$ to $+20 \%$. (b) Quality factor sensitivity $\widetilde{Q}$-map; values are ranging from $-60 \%$ to $+74 \%$, the two displayed isolines are at $-20 \%$ and $+20 \%$. (c) Mean drag change displayed as $\widetilde{C}_{D}$-map; the interval between the isolines is $5 \%$ with values ranging from $-45 \%$ to $+8 \%$. (d) The rate of fluctuation of drag $\widetilde{\delta}_{D^{-}}$-map; values are ranging from $-90 \%$ to $+350 \%$, the four displayed isolines are at $-50 \%$, $-20 \%,+30 \%$, and $+150 \%$. Numbered positions correspond to the flow configurations, as shown in Fig. 3. 

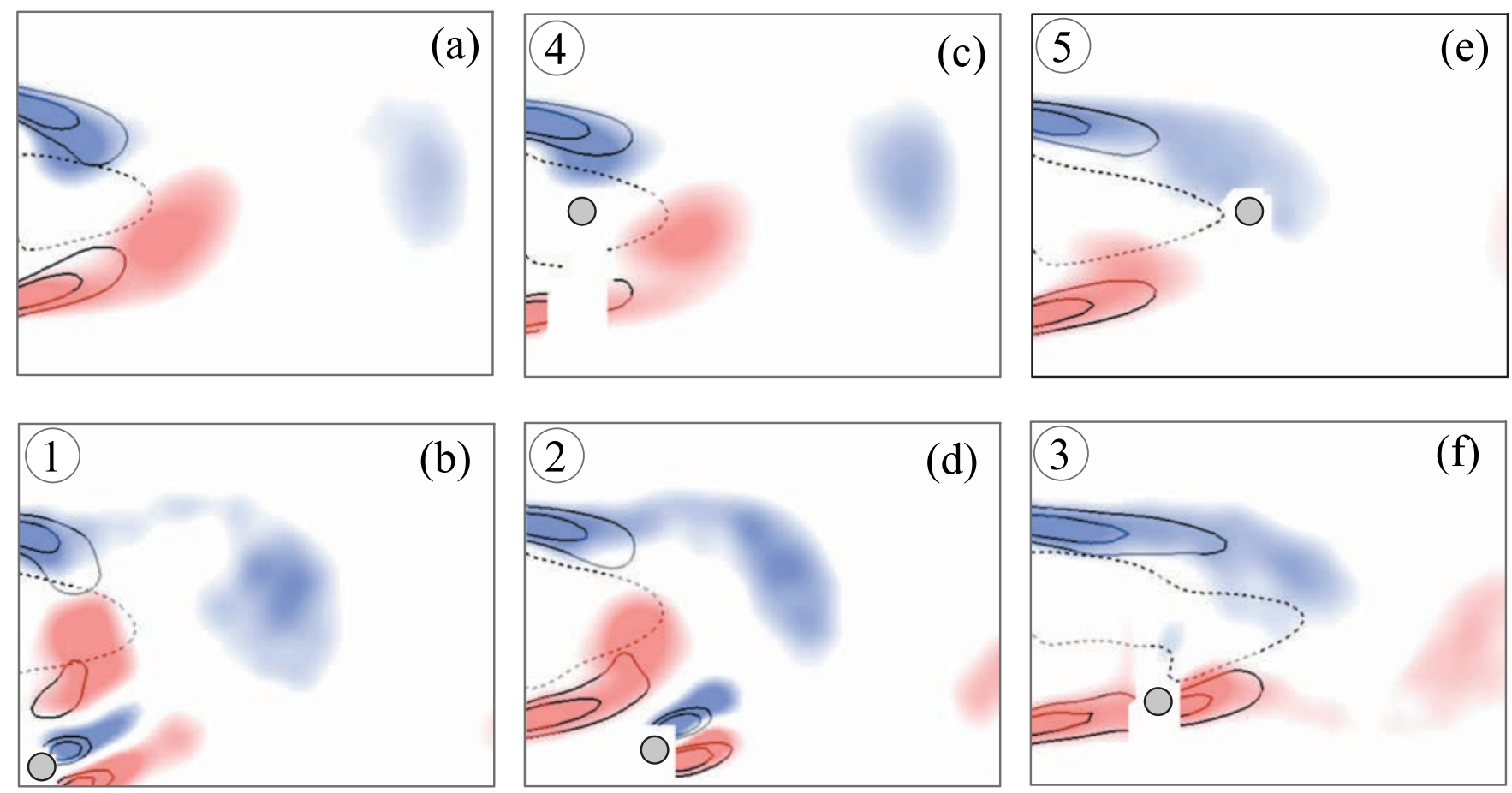

FIG. 3. (Color) PIV results showing the instantaneous vorticity, the mean vorticity, and the backflow region for configurations 1-5 referenced in Fig. 2. Color maps display phase-averaged vorticity, with red for positive and blue for negative vorticity. The continuous lines are isolines of the mean vorticity at \pm 2000 and $\pm 3000 \mathrm{~s}^{-1}$. The dashed line is the zero isoline of the mean longitudinal velocity. The region inside the dashed line contains the backflow and its total length corresponds to the recirculation bubble length. (a) shows the flow without the control cylinder; the dimensionless coordinates $\left(x_{C} / D, y_{C} / D\right)$ of various configurations, when the control cylinder is present, are (b) $(0.12,-0.76),(\mathrm{c})(0.44,0),(\mathrm{d})(0.78,0.68),(\mathrm{e})(1.26,0)$, and (f) $(0.78,0.4)$.

toward the large velocity in the shear layers. ${ }^{9}$ When the control cylinder is at the inner edge of the shear, as in Fig. 3(f), the entrained velocity forces the shear to stick to the control cylinder. If so, the global frequency can only increase due to constructive vorticity interaction. The sheet reattachment on the control cylinder allows the separated region to be extended downstream [note the increase in the backflow region length in Fig. 3(f)]. The frequency increase in the region around location 2 may be interpreted by flow deviation. In this case, the deviation of the flow by the control cylinder pushes the primary vortex sheet [Fig. 3(d)] without direct vorticity interaction. The blockage due to the presence of the control cylinder accelerates the flow and changes the vorticity distribution in the shear. For location 4 , where the frequency is increased, the PIV measurements [Fig. 3(c)] show that the control cylinder is in the middle of the backflow region. Here the mechanism of frequency increase involves a periodic feedback effect. The vorticity created by the control cylinder is convected upstream and periodically affects the primary vortex sheet before the rolling-up process. When the distance $x_{C}$ is increased downstream, the frequency reduces gradually to its natural value; however, major effects are still observable on the mean flow, as, for instance, in configuration 5 , for which $\tilde{f} \approx 0$, the bubble is stretched due to a flow reattachment on the control cylinder [Fig. 3(e)]. For positions of the control cylinder further downstream, the mean bubble shrinks gradually to its natural size.

As for sensitivity analysis obtained around the critical Reynolds number, ${ }^{1,5,6}$ the global mode frequency selection is found to be sensitive to perturbations inside the bubble (lo- cation 4), in and around the shears (location 1 and 3), and outside the bubble in location 2 . On the other hand, only reductions in frequency are found in Refs. 1, 5, and 6, while both increases and decreases are found in the present experiment. Near the threshold of the instability, the role of the perturbation, whatever its position is, is to stabilize the instability ${ }^{5}$ and the main resulting effect reduces the frequency. For larger Reynolds numbers, one may wonder what a theoretical sensitivity analysis would predict if performed on the mean flow as done in Refs. 10 and 11 for the natural wake.

We now turn to the sharpness of the flow synchronization measured by the quality factor. It is shown by the $\widetilde{Q}$-map in Fig. 2(b). The quality factor is $60 \%$ reduced in the blue regions and $74 \%$ increased in the red ones. The reattached flow configurations 3 and 5 have a small quality factor certainly because the reattachment to a fixed point inhibits oscillations of the velocity field. The quality is also reduced when the control cylinder is right in the middle of the bubble (configuration 4), where the vorticity feedback effect occurs and also, but less pronounced, in the two symmetric regions located at $y_{C}= \pm 0.33 D$ in the vicinity of the rear of the main body. Except for these three positions, $\widetilde{Q}$ is increased when the control cylinder is in the recirculation bubble.

Consequences on the form drag are drastic as we can notice in the $\widetilde{C}_{D^{-}}$-map [Fig. 2(c)]. The larger drag reduction (up to $45 \%$ ) is associated with the interaction of the control cylinder with the primary shear layers (two symmetric streaks around location 3). For these regions, the roll-up pro- 
cess takes place far downstream causing an increase in the recirculation bubble length [Fig. 3(f)]. A similar effect is observable around the position $y_{C}=0, x_{C}=1.26 D$ [see configuration 5 in Fig. 3(e)], where the drag is also reduced. The downstream displacement of the roll-up region together with the increase in the bubble length increases the base pressure which reduces the drag. ${ }^{12}$ We can notice two symmetric regions that correspond to local drag reduction maxima around location 2. As said before, the acceleration associated with the flow deviation might also increase the bubble length. Finally, we can observe two regions of small, but nevertheless significant drag increase of $8 \%$ when the control cylinder is right in the middle of one of the two eddies constituting the recirculation bubble.

The drag fluctuation rate shown in the $\widetilde{\delta}_{D}$-map in Fig. 2(d) is mostly reduced except in much localized regions. For those regions (around location 5 and beside location 3), the high speed PIV shows that the recirculation bubble alternates between two states; it can either be attached to the control cylinder, having a large size with low drag, or detached with its much smaller natural size and high drag. This alternative creates a strong crisis on the drag fluctuations. For the other blue regions, the reduction in the fluctuation rate is strongly correlated with the reduction in the mean drag. The lower the drag, the larger is the bubble size, causing the unsteady roll-up process to take place further downstream. For large bubble lengths, the near wake of the main body simply becomes less unsteady.

In conclusion, the global properties of a wake at large Reynolds number are sensitive to a perturbation located in and around the shear layers or inside the recirculation bubble. This is in qualitative agreement with both previous theoretical and experimental sensitivity analyses at low Reynolds number. However, there is a disagreement with the frequency selection. A theoretical approach based on the mean flow stability analysis might be more relevant for applications at large Reynolds number. From our PIV measurements, the global mode frequency selection is attributed to vorticity interaction between the control cylinder and the pri- mary shear layers. The flow reattachment on the control cylinder can drastically increase the recirculation bubble size, which could explain the origin of mean and fluctuating drag modifications. Our experimental work creates avenues to other studies, especially about the selection of the quality factor of the shedding mode displayed in Fig. 2(b). The quality factor is intimately related to the three-dimensional properties of the wake ${ }^{13}$ that we will further investigate.

This work was supported by the ANR Project No. 06BLAN-0363-01 "HighSpeedPIV."

${ }^{1}$ P. J. Strykowski and K. R. Sreenivasan, "On the formation and suppression of vortex shedding at low Reynolds number," J. Fluid Mech. 218, 71 (1990).

${ }^{2}$ D.-C. Hill, "A theoretical approach for analyzing the restabilization of wakes," AIAA Paper No. 92-0067 (1992).

${ }^{3}$ J.-M. Chomaz, "Global instabilities in spatially developing flows: Nonnormality and nonlinearity," Annu. Rev. Fluid Mech. 37, 357 (2005).

${ }^{4}$ F. Giannetti and P. Luchini, "Structural sensitivity to the first instability of the cylinder wake," J. Fluid Mech. 581, 167 (2007).

${ }^{5}$ O. Marquet, D. Sipp, and L. Jacquin, "Sensitivity analysis and passive control of cylinder flow," J. Fluid Mech. 615, 221 (2008).

${ }^{6}$ P. Luchini, F. Giannetti, and J. Pralits, "Structural sensitivity of the finiteamplitude vortex shedding behind a circular cylinder," in Proceedings of the IUTAM Symposium on Unsteady Separated Flows and Their Control, edited by M. Brazza and K. Hourigan (Springer, Dordrecht, 2009), Vol. 14, pp. 131-140.

${ }^{7}$ H. Sakamoto, K. Tan, and H. Haniu, "An optimum suppression of fluid forces by controlling a shear layer separated from a square prism," ASME Trans. J. Fluids Eng. 113, 183 (1991).

${ }^{8}$ B. Thiria, O. Cadot, and J.-F. Beaudoin, "Passive drag control of a blunt trailing edged cylinder," J. Fluids Struct. (in press).

${ }^{9}$ J. H. Gerrard, "The mechanics of the formation region of vortices behind bluff bodies," J. Fluid Mech. 25, 401 (1966).

${ }^{10}$ D. Barkley, "Linear analysis of the cylinder wake mean flow," Europhys. Lett. 75, 750 (2006).

${ }^{11}$ D. Sipp and A. Lebedev, "Global stability of base and mean flows: A general approach and its applications to cylinder and cavity flows," J. Fluid Mech. 593, 333 (2007).

${ }^{12}$ A. Roshko, "Perspectives on bluff body aerodynamics," J. Wind. Eng. Ind. Aerodyn. 49, 79 (1993).

${ }^{13} \mathrm{C}$. H. K. Williamson, "The natural and forced formation of spot-like "vortex dislocations' in the transition of a wake," J. Fluid Mech. 243, 393 (1992). 